\title{
Combination immunotherapy in metastatic renal cell carcinoma. Are we leaving something back?
}

\author{
Vincenzo Di Nunno', Lidia Gatto², Benedetta Fragomeno', Marta Cubelli', Elisabetta \\ Nobili' ${ }^{1}$, Ida Romano ${ }^{1}$, Matteo Santoni ${ }^{3}$, Salvatore Pisconti ${ }^{2}$, Rodolfo Montironi ${ }^{4}$ \& \\ Francesco Massari*,1 \\ ${ }^{1}$ Division of Oncology, S Orsola-Malpighi Hospital, Bologna, Italy \\ ${ }^{2}$ Oncology Unit, SG Moscati Hospital of Taranto, Taranto, Italy \\ ${ }^{3}$ Oncology Unit, Macerata Hospital, via Santa Lucia 2, Macerata, Italy \\ ${ }^{4}$ Section of Pathological Anatomy, School of Medicine, United Hospital, Polytechnic University of the Marche Region, Ancona, Italy \\ *Author for correspondence: fmassari79@gmail.com
}
"The immune-revolution is far from being finished and new clinical challenges await an answer in the coming years"

First draft submitted: 9 August 2018; Accepted for publication: 12 September 2018; Published online: 9 November 2018

Keywords: clinical trials $\bullet$ immunotherapy $\bullet$ metastatic renal cell carcinoma

In Checkmate214, Motzer et al. demonstrated a significant clinical benefit in terms of overall survival (OS) and overall response rate (ORR) for previously untreated intermediate/poor risk patients with metastatic renal cell carcinoma (RCC) receiving nivolumab/ipilimumab combination over sunitinib [1].

Results of this Phase III trial have led to a revolution in the management of metastatic clear cell RCC (ccRCC) since for the first time a combination of agents deeply different from angiogenesis inhibitors has showed to improve patient's clinical outcomes in the first-line setting.

As observed in melanoma [2,3] and more recently in non-small-cell lung cancer (NSCLC) [4], the synergic effect resulting from a combination of immune-checkpoint (PD-1 and CTLA-4) inhibitors has led to impressive results also in ccRCC.

The immune-revolution is far from being finished and new clinical challenges await an answer in the coming years [5,6]. Our goal is to obtain longer remission and better clinical benefit for RCC patients, our risk is to deny this benefit to some of them.

Thus, one of the most important issues is focused on the selection of patients more likely to respond to immune-checkpoint inhibitors.

In Checkmate214, we observed that immune-checkpoint clinical activities increase with increasing tumor aggressively like PD-L1 expression. However, this latter has failed to show a significant predictive response value. This particular behavior leads to hypothesize that increasing tumor aggressiveness could be associated with the acquisition of a higher mutation load reflecting in higher neo-antigens expression that are related to better immunotherapy outcomes [7-9].

The development of higher mutation burden seems to be a feature of more aggressive tumors (such as intermediate/poor risks) and, to reinforce this hypothesis, the nivolumab-ipilimumab combination has recently shown to increase response rate and progression-free survival of patients with stage IV NSCLC with high mutational burden. It would be plausible to suppose a correlation between mutational load and MSKCC or IMDC prognostic criteria as the higher mutational load the poorer patients risk categories. However, this relationship has not been demonstrated for prognostic criteria as well as for other biological features associated to poor prognosis. Indeed, no mutational load rate difference between sarcomatoid component and clear cell component has been observed [10,11]. Recently, de Velasco et al. performed a whole exomes and transcriptomes sequencing of nine mRCC patients treated with nivolumab. Overall they found a few number of nonsynonymous mutations. On the 
contrary of what was expected, nonresponder patients (six) showed a higher nonsynonymous (and neo-antigens) rate compared with responder patients (three) [12]. Of course no definitive conclusions could be obtained from this study, mainly for the small number of patients analyzed. However, the role of nonsynonymous mutations in RCC is not well explored and should be better investigated in further clinical trials. In addition, it is worth mentioning that even if combination therapy failed to show a significant ORR improvement in favorable risk RCC patients, the complete response rate obtained in this subgroup was 11 versus $6 \%$ with sunitinib. So it is possible that a subgroup of patients with favorable risk profile could hide a not negligible sensitivity to immune-checkpoint inhibitors.

Therefore, the next challenge is the development of treatment strategies able to increase clinical benefit of immune-checkpoint inhibitors and to stretch this benefit to a large population of patients. Leading on from this, one of the most important approaches being studied employs the combination of immune-checkpoint inhibitors and a target agent. Angiogenesis and immunosuppression are strategies commonly adopted by tumors to acquire treatment resistance and promote their growth and progression. However, further evidence seems to indicate a deep correlation between these two pathways. Indeed, VEGF/VEGFR inhibition results in an increased lymphocyte PD-L1 expression. The blockade of VEGFR and PD-L1 pathways induces high endothelial venules that promote lymphocyte infiltration and activity through activation of lymphotoxin receptor [14,15]. VEGF could also inhibit dendritic cell maturation and leukocyte-endothelial interaction by reducing ICAM-1 and VCAM1 adhesion molecules [16]. The combination between axitinib (a VEGFR 1,2,3 inhibitor) and pembrolizumab (a PD-1 inhibitor) has been tested in a Phase IB trial showing a very promising ORR (73\%) with a tolerable safety profile $[17,18]$. Bevacizumab and tezolizumab combination has been evaluated in a Phase II trial (IMmotion 150) and showed an encouraging antitumor activity [19] justifying the planning of a Phase III trial (IMmotion 151) [20]. In this last trial, treatment-naive patients with metastatic RCC were randomized to receive atezolizumabbevacizumab combination or sunitinib. Median survival follow-up was 15 months and OS was immature at first interim analysis. However, the combination showed a better PFS and ORR compared with sunitinib. Moreover, in PD-L1+ patients, ORR was $43 \%$ and duration of response was not reached for atezolizumab + bevacizumab versus $35 \%$ and 12.9 months for sunitinib.

On the wave of these surprising results, several large Phase III trials are evaluating a combination between an immune-checkpoint inhibitor and a target agent. Nivolumab-cabozantinib (CheckMate 9ERNCT03141177), pembrolizumab-axitinib (KEYNOTE 426-NCT02853331) and lenvatinib-pembrolizumab (CLEAR-NCT02811861) combination are currently under study.

To date, we do not have sufficient data about the clinical impact of these combinations and a comparison between immune-checkpoints (CTLA4/PD-1) and immune-target combination is still unavailable. However, the attractive multi-pathway inhibition obtained by immune-target combination leads to speculating that these approaches could result in a clinical benefit obtainable from a larger population than observed in Checkmate214. On the other hand, it would also be important to evaluate if the combination of more than a sequencing approach results in a survival and clinical improvement.

Combination strategies could be a valid option also in an advanced setting of the disease. As known, nivolumab is an active treatment that showed to improve overall survival of patients progressed to standard first-line treatment in Checkmate025 trial [5]. Do we have strategies that are able to improve clinical benefit of patients receiving immunotherapy in this setting?

In particular, we do not know if the addition of ipilimumab to nivolumab as a salvage therapy could result in better clinical outcome in advanced lines treatment. The results from a Phase II trial (NCT03117309) evaluating nivolumab as front-line treatment reserving the addition of ipilimumab in patients with symptomatic and/or confirmed progressive disease could be of particular interest due to their potential application to more advanced lines of treatment.

In conclusion, we are going through a very exciting era for the management of metastatic RCC. Clinical impact of immune-target combinations/sequences; assessment of the better approach between immune checkpoint inhibitors or immune-target combinations; development of reliable predictive response markers and evaluation of the role of these approaches in advanced setting of the disease, as well as in specific patient's population (such as patients with nonclear cell RCC), are important issues that should be solved in the coming years.

Immunotherapy represents a concrete hope for RCC patients; our challenge is the development of strategies able to extend the number of patients that can have more to benefit more by these treatments. 


\section{Financial \& competing interests disclosure}

The authors have no relevant affiliations or financial involvement with any organization or entity with a financial interest in or financial conflict with the subject matter or materials discussed in the manuscript. This includes employment, consultancies, honoraria, stock ownership or options, expert testimony, grants or patents received or pending, or royalties.

No writing assistance was utilized in the production of this manuscript.

\section{References}

1 Motzer RJ, Tannir NM, McDermott DF et al. Nivolumab plus ipilimumab versus sunitinib in advanced renal-cell carcinoma. N. Engl. J. Med. 378(14), 1277-1290 (2018)

2 Wolchok JD, Chiarion-Sileni V, Gonzalez R et al. Overall survival with combined nivolumab and opilimumab in advanced melanoma. N. Engl. J. Med. 377(14), 1345-1356 (2017).

3 Weber J, Mandala M, Del Vecchio M et al. Adjuvant nivolumab versus ipilimumab in resected stage III or IV melanoma. N. Engl. J. Med. 377(19), 1824-1835 (2017).

4 Hellmann MD, Ciuleanu TE, Pluzanski A et al. Nivolumab plus ipilimumab in lung cancer with a high tumor mutational burden. $N$. Engl. J. Med. 378(22), 2093-2104 (2018).

5 Ciccarese C, Di Nunno V, Iacovelli R, Massari F. Future perspectives for personalized immunotherapy in renal cell carcinoma. Expert Opin. Biol. Ther. 17(9), 1049-1052 (2017).

6 Santoni M, Massari F, Di Nunno V et al. Immunotherapy in renal cell carcinoma: latest evidence and clinical implications. Drugs Context 7, 212528 (2018).

7 Motzer RJ, Escudier B, McDermott DF et al. Nivolumab versus everolimus in advanced renal-cell carcinoma. N. Engl. J. Med. 373(19), 1803-1813 (2015).

8 Rizvi NA, Hellmann MD, Snyder A et al. Cancer immunology. Mutational landscape determines sensitivity to PD-1 blockade in non-small cell lung cancer. Science 348(6230), 124-128 (2015).

9 Yarchoan M, Hopkins A, Jaffee EM. Tumor mutational burden and response rate to PD-1 inhibition. N. Engl. J. Med. 377(25), 2500-2501 (2017).

10 de Velasco G, Miao D, Voss MH et al. Tumor mutational load and immune parameters across metastatic renal cell carcinoma risk groups. Cancer Immunol. Res. 4(10), 820-822 (2016).

11 Malouf GG, Ali SM, Wang K et al. Genomic characterization of renal cell carcinoma with sarcomatoid dedifferentiation pinpoints recurrent genomic alterations. Eur. Urol. 70(2), 348-357 (2016).

12 De Velasco G, Miao D, Shukla S. Integrated genomic correlates of response to PD-1 inhibitor nivolumab in metastatic renal cell carcinoma (mRCC). J. Clin. Oncol. 34(Suppl. 2S), Abstract 545 (2016).

13 Allen E, Jabouille A, Rivera LB et al. Combined antiangiogenic and anti-PD-L1 therapy stimulates tumor immunity through HEV formation. Sci. Transl. Med. 9, 385 (2017).

14 Zappasodi R, Merghoub T, Wolchok JD. Emerging concepts for immune checkpoint blockade-based combination therapies. Cancer Cell 33(4), 581-598 (2018)

15 Dirkx AE, Oude Egbrink MG, Kuijpers MJ et al. Tumor angiogenesis modulates leukocyte-vessel wall interactions in vivo by reducing endothelial adhesion molecule expression. Cancer Res. 63(9), 2322-2329 (2003).

16 Kandalaft LE, Motz GT, Busch J, Coukos G. Angiogenesis and the tumor vasculature as antitumor immune modulators: the role of vascular endothelial growth factor and endothelin. Curr. Top Microbiol. Immunol. 344, 129-148 (2011).

17 Atkins MB, Plimack ER, Puzanov I et al. Axitinib in combination with pembrolizumab in patients with advanced renal cell cancer: a non-randomised, open-label, dose-finding, and dose-expansion Phase 1b trial. Lancet Oncol. 19(3), 405-415 (2018).

18 Di Nunno V, Santoni M, Massari F. Re: Michael B. Atkins, Elizabeth R. Plimack, Igor Puzanov, et al. Axitinib in combination with pembrolizumab in patients with advanced renal cell cancer: a non-randomised, open-label, dose-finding, and dose-expansion Phase $1 \mathrm{~b}$ trial. Lancet Oncol 2018;19:405-15. Eur. Urol. 74(2), e50 (2018).

19 Atkins MB, McDermott DF, Powles T et al. IMmotion150: a Phase II trial in untreated metastatic renal cell carcinoma (mRCC) patients (pts) of atezolizumab (atezo) and bevacizumab (bev) vs and following atezo or sunitinib (sun). J. Clin. Oncol. 35(Suppl.

15), 4505-4505 (2017).

20 Motzer RJ, Powles T, Atkins MB et al. IMmotion151: a randomized Phase III study of atezolizumab plus bevacizumab vs sunitinib in untreated metastatic renal cell carcinoma (mRCC). J. Clin. Oncol. 36(Suppl. 6), 578-578 (2018). 
